\title{
PRONÓSTICO DE TSUNAMIS PARA LAS ISLAS GALÁPAGOS
}

Willington Rentería ${ }^{1}$

\section{RESUMEN}

Este estudio presenta la implementación de un sistema de Pronóstico de Tsunamis para las Islas Galápagos. Este sistema, consiste en el desarrollo de modelos de pronóstico de tsunamis de corto y de largo plazo. El primero es utilizado en tiempo real, una vez que se conozca la ocurrencia de un tsunami en el Océano Pacífico, a fin de conocer los efectos físicos que el evento producirá a su impacto con las Islas. Mientras que el pronóstico de largo plazo, servirá para identificar las zonas de generación de tsunamis de potencial peligro para las islas, así como también, tener una referencia inmediata para la evaluación de un evento en particular.

El modelo numérico utilizado para la ejecución de ambos pronósticos es el MOST(Method of splitting Tsunami)[1] con la metodología ComMIT(Community Model Interface for Tsunami)[2], la metodología ha sido adaptada a las condiciones particulares locales.

Keywords: pronóstico, tsunamis, Galápagos

\begin{abstract}
This study present the implementation of a Tsunami forecast system for Galapagos Islands. This system is formed by the development of short and long term forecast models. The first is used in real time, with the information of the ocurrence of a tsunami event in the Pacific Ocean, in order to predict the physical effects of the impact on islands. While , the long term forecast, is used to identify tsunami generation zones with potential threat to damage the islands and also is used to have a reference to evaluate an particular event.
\end{abstract}

The numerical model used to get both forecasts is MOST(Method of splitting Tsunamis) with ComMIT methodology (Community Model Interface for Tsunami), which has been adapted to local conditions. 


\section{INTRODUCCIÓN}

Debido a su ubicación geográfica las Islas Galápagos se encuentran en un alto nivel de exposición a los tsunamis que suceden en el Océano Pacífico. A pesar de no tener una fuente cercana para la generación de sismo-tsunamis, su localización hace que se encuentre en el paso obligado de los tsunamis que transitan por este océano. Su nivel de exposición es distinto al de la costa ecuatoriana, que tiene la zona de subducción a unos cuantos kilómetros, Galápagos por su parte posee una configuración litoral omnidireccional, lo que expone a sus poblaciones costeras a las diferentes amenazas tsunamigenicas, provenientes de las varias zonas de subducción del Pacífico.

Para responder a la amenaza de tsunamis, tanto para Galápagos como para las provincias costeras, el Ecuador es parte del Sistema de Alerta de Tsunamis del Pacífico, cuyo punto focal es el Instituto Oceanográfico de la Armada, con su Centro Nacional de Alerta de Tsunamis. Este Centro recomendó al Gobierno las acciones a seguir, luego de los tsunamis de Febrero 28, 2010 y Marzo 11, 2011; generador por sismo de magnitudes 8.8 y 9.0 , respectivamente en la escala de momento sísmico. En ambos casos, esta recomendación se basó en la aplicación de Procedimientos Operativos Standard, los que permiten una rápida evaluación del evento en función de la localización del epicentro, magnitud del sismo y profundidad del hipocentro, principalmente. Esta información es reforzada con el análisis de eventos históricos en la zona sismo-generadora, la distancia al epicentro del evento sísmico y el tiempo de arribo de las olas generadas por el tsunami. Todas estas variables forman parte de una matriz de decisión, que se concreta al momento de evaluar el nivel de amenaza que presenta un evento tsunami-génico al generarse. Sin embargo, aunque este proceso garantiza una evaluación objetiva del evento, permitiendo en primera instancia alertar o no a la población; este proceso no entrega información respecto a los posibles efectos que el tsunami pueda generar al momento de arribar a las costas. Este tipo de información se puede conseguir solamente a través de una simulación del evento y con la sistematización de datos que permita un pronóstico operacional de tsunamis.

La necesidad de herramientas operacionales que produzcan un adecuado pronóstico de tsunamis y que permitan una rápida evaluación de los eventos, ha sido discutida ampliamente en[3], donde se realiza un análisis prospectivo del estado de los sistemas de pronóstico de tsunami existentes, los retos y cambios en los modelos vigentes. La razón por la que esta información es importante, es porque permite una cuantificación de los datos de la máxima distancia de inundación, la altura de flujo, y la velocidad de las corrientes. Con esta información disponible al menor tiempo posible, las autoridades competentes pueden organizar rápidamente la respuesta para hacer frente a la situación.

El presente estudio tiene por objeto presentar una propuesta de pronóstico operacional de Tsunamis para las Islas Galápagos, el mismo que incluye la generación de modelos de inundación para tres diferentes lugares de las islas, y que pueden ser utilizados en el corto plazo, es decir, inmediatamente luego de ocurrido del tsunami a fin de conocer cuantitativamente los efectos del mismo. Así mismo, se presenta la generación de pronóstico de tsunamis pre-computados, para 103 zonas sismo-generadoras, que para fines de gestión de riesgos, pueden ser utilizadas para identificar cuales serian las zonas que presenten una amenaza potencial una vez que produzcan un tsunami.

La base de esta propuesta será el empleo de la herramienta ComMIT/MOST, cuya metodología será explicada de manera sucinta en este documento. Los resultados de la propuesta de corto plazo, son validados con los datos levantados de los efectos del tsunami de Tohoku en las costas de Galápagos; además se expondrá la utilización de los resultados del pronóstico de largo plazo y su uso en el Centro de Investigaciones Marinas Galápagos. 


\section{METODOLOGÍA COMMIT/MOST}

\section{A. Modelo numérico MOST}

A continuación se realizará una descripción comprensiva del modelo, que es la base para el modelamiento numérico de la presente propuesta, sin embargo, mayor información se puede encontrar en[4],[5].

El modelo MOST(Method of Splitting Tsunamis) se deriva del modelo VTCS [4] y su formulación matemática consiste en convertir las ecuaciones bidimensionales no-lineales de aguas someras integradas en la vertical, en dos sistemas de ecuaciones hiperbólicas, para las dimensiones espaciales en el plano horizontal, esta conversión se realiza usando el método de pasos fraccionales[6]. MOST obedece a esta formulación matemática para la simulación de la propagación del tsunami, sin embargo el código también incluye la simulación de las etapas de generación del tsunami e inundación o impacto costero.

MOST, consiste en tres etapas, la primera que es la simulación del proceso de generación y se basa en el modelo de plano de falla de la fuente sismo-generadora desarrollado por Okada [7], y asume la incomprensibilidad de la columna de agua ubicada sobre el plano de falla. MOST calcula las condiciones iniciales de perturbación en base a esa deformación estática del suelo marino. La principal asumpción, es que esta deformación ocurre tan rápidamente que la perturbación en la superficie del mar es un reflejo de lo que ocurre al nivel del suelo marino, ya que la velocidad horizontal de la propagación de la perturbación es mucho menor que la velocidad con la que se transfiere la perturbación en la vertical.

En la segunda etapa, el modelo simula la propagación de las ondas, aplicando un sistema de grillas anidadas. Aquí el modelo MOST, utiliza la formulación matemática de la separación de las ecuaciones y las discretiza utilizando el método de diferencias finitas en un esquema explícito, con una aproximación de segundo y primer orden, en espacio y tiempo respectivamente. Con estas ecuaciones se computa los valores de las invariantes de Riemann, obtenidas luego de la aplicación de los pasos fraccionales y que se utilizan para calcular los valores de $\mathrm{U}, \mathrm{V} \mathrm{y} \mathrm{H}$, que son respectivamente el componente de velocidad en $\mathrm{X}$, en $\mathrm{Y}$ y la perturbación del nivel del mar. Así mismo, este modelo ha sido probado para diferentes eventos [8],[9].

La última etapa es la de inundación, para esta se necesita convertir el borde del dominio computacional en uno dinámico; en esta fase, el modelo aplica un algoritmo que de acuerdo a la relación tiempo/espacio proyecta el avance de la ola sobre el perfil de la costa, utilizando el valor del último nodo dentro del dominio computacional, y de esta manera ir incrementado el borde tierra adentro. En esta etapa se busca calcular el "run-up", en base a la altura alcanzada por las aguas y la distancia inundada desde el límite del borde en las condiciones iniciales.

Cabe destacar que el cálculo de "run-up", es un campo activo de la investigación[10] del comportamiento de las olas al momento de arribar a las costas. El grado de dificultad al momento de calcularlo se debe al incremento de los efectos no lineales provocados por el cambio del fondo marino (batimetría) tanto en la vertical como en la horizontal. Una buena referencia respecto al calculo del runup se puede encontrar en [11].

\section{B. Metodología ComMIT/MOST}

El ComMIT/MOST es una iniciativa del PMEL/NOAA( Pacific Marine Environmental Laboratory/ National Ocean Atmosphere Agency) para implementar una comunidad de intercambio en el modelamiento de tsunamis [2]. Su objetivo es proveer de una plataforma con una interfaz grafica intuitiva para el modelamiento numérico de tsunamis. Esta interfaz gráfica permite que de una manera sencilla que se ingresen los parámetros que necesita el modelo MOST para la simulación del Tsunami. ComMIT/MOST fue pensado para ser empleado por personal entrenado[2], sin necesidad de ser científico experto en tsunamis. 
ComMIT/MOST se basa en el empleo de la herramienta operacional SIFT(Short-term Inundation Forecast for Tsunami), y de SIMs (Standby Inundation Models). El SIFT consiste en escenarios de propagación de tsunamis precomputados, ligados a una fuente generadora conocida, cuyos parámetros y ubicación han sido escogidos para garantizar la generación de un tsunami, tomando como referencia la historia sísmica de cada sitio[12], estas reciben el nombre de unidades fuentes. Los SIFTs han sido almacenados en bases de datos y son extraídos conforme la necesidad de simular eventos particulares de acuerdo a cada región. Mientras que los SIMs, consisten en un conjunto de grillas que forman parte del dominio computacional, en el que se desarrollará la simulación; estas grillas deben cumplir ciertos requisitos a fin de garantizar el menor tiempo de ejecución de los modelos, para lo cual se debe armonizar entre las dimensiones de cada grilla, la resolución y el tiempo en el que se quiere obtener los resultados.

El empleo del ComMIT/MOST, de los SIFTs y los SIMs, consiste en un sistema operacional que permite el modelamiento rápido del tsunami. El uso del usuario final se puede dar en dos diferentes modos: manual y con solución numérica. En el modo manual, de acuerdo a los parámetros del sismo, el usuario escoge las unidades fuentes e ingresa los parámetros para la combinación lineal de las mismas. Esta combinación produce las condiciones iniciales y de borde dinámico que son empleadas por el modelo MOST para la simulación del tsunami en el área escogida a través de los SIMs. Con solución numérica, los parámetros de combinación y las unidades fuentes necesarias, se obtienen refinando la solución manual utilizando los datos de la boya DART[9].

Ambas soluciones se basan en que la propagación del tsunami en el océano profundo es mayormente lineal[13], ya que la amplitud de las olas es muy pequeña en comparación con la longitud de onda, de esta manera la soluciones pre-computadas de los SIFTs pueden combinarse linealmente para simular un tsunami que resulte de la combinación de las unidades fuentes.

$$
\text { (1) } T_{M}=\mu L W \sum_{1}^{k} u_{k}
$$

Las unidades fuente que se combinan, utilizan los parámetros conocidos $\mu, \mathrm{L}, \mathrm{W}$ que son el coeficiente de rigidez, la longitud y ancho del plano de falla, respectivamente; uk, representa el slip que se necesita aplicar a cada unidad fuente para simular un sismo-tsunami, con la magnitud similar al evento que quiere modelarse.

Como se mencionó anteriormente, en el proceso manual los valores del slip, son escogidos manualmente empleando (1) hasta conseguir un magnitud de tsunami que represente su contraparte de magnitud de momento sísmico, para lo cual se emplea la relación $\mathrm{Mw}=(2 / 3) \log (\mathrm{Mo})$.

En la solución numérica, se emplea un algoritmo inverso, que utiliza series de tiempo extraídas de cada unidad fuente $g(t) j, k$, en la posición de la boya DART, para combinarlas linealmente y obtener una serie de tiempo similar a la obtenida en tiempo real por la boya DART, $d(t) j$.

(2)

$$
d(t)_{j}=\alpha_{1} g(t)_{j, 1}+\ldots+\alpha_{k} g(t)_{j, k}+e_{j}
$$

Los coeficientes para la combinación lineal se obtienen a partir de la obtención del error mínimo cuadrado, entre la serie de tiempo real y las series de tiempo pre-computada usadas para la solución. Este algoritmo se encuentra ampliamente explicado en [9].

De esta manera se consigues las condiciones iniciales y de borde dinámico para la simulación de eventos en las comunidades costeras. Con esto se obtiene una aproximación del tiempo de arribo y altura de ola, así como su inundación, información que como se mencionó antes permitirá una rápida organización de la respuesta.

\section{EFECTOS DEL TSUNAMI TOHOKU JAPÓN 2011, EN LAS COSTAS DE LAS ISLAS GALÁPAGOS}

En marzo 11, a las 05:46 UT ocurrió un sismo de magnitud 9.0, el cual produjo un tsunami que azotó varias localidades a largo de las costas del Océano Pacifico. Tras este tsunami el Estado Ecuatoriano emitió una alerta y orden de evacuación para todas sus costas y región insular. Adicionalmente se dispuso la paralización de las actividades marítimas, obligando además el 
desvío del tráfico marítimo y la prohibición de ingresar a las bahías y puertos de Galápagos.
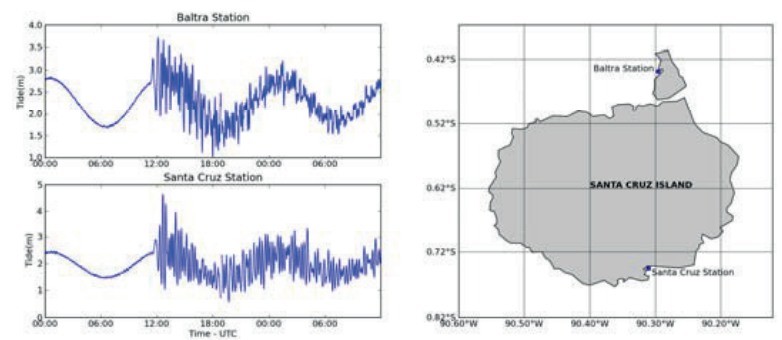

Figura 1 Registro mareográfico del tsunami de Japón, Marzo 2011, en Puerto Ayora-Isla Santa Cruz y Puerto Seymour-Isla Baltra.

Este tsunami fue registrado por dos mareógrafos y un correntómetro en las Islas Galápagos[14], [15]. El impacto del tsunami en las islas dejó grandes afectaciones a la ciudad de Puerto Ayora en Santa Cruz, Galápagos, la misma que fue la más afectada de los lugares poblados en las islas. Aunque se tiene el registro del mareógrafo de Puerto Ayora, este sitio no fue mayormente afectado.

El registro mareográfico del arribo de las olas (Figura 1), muestra que las perturbaciones se mantuvieron persistentes por aproximadamente tres días, hasta que la superficie del mar retornó a su estado habitual.

En los días posteriores al tsunami, Lynett et al.,(2011) realizaron un levantamiento de los impactos del tsunami sobre las costas de Galápagos; de este estudio se han extraído los datos levantados de los sitios que serán utilizados en el presente estudio. Los datos extraídos se registran en la Tabla 1.

\begin{tabular}{|l|c|r|r|}
\hline \multicolumn{1}{|c|}{ Sitio } & Coordenadas & $\begin{array}{c}\text { Run- } \\
\text { up } \\
(\mathbf{m})\end{array}$ & $\begin{array}{c}\text { Inund. } \\
\text { (m) }\end{array}$ \\
\hline Pto. Ayora & S0 44.807 W90 18.696 & 2.81 & 40 \\
\hline Pto. Ayora & S0 44.834 W90 18.756 & 2.44 & 0 \\
\hline Pto. Ayora & S0 44.561 W90 18.589 & 2.56 & 23.4 \\
\hline Pto. Ayora & S0 44.571 W90 18.672 & 2.01 & 129.5 \\
\hline Pto. Ayora & S0 44.657 W90 18.723 & 2.69 & 25 \\
\hline Pto. Baquerizo & S0 54.084 W89 36.702 & 2.16 & 46.9 \\
\hline Pto. Baquerizo & S0 54.138 W89 36.829 & 2.23 & 25 \\
\hline Pto. Baquerizo & S0 54.116 W89 36.836 & 2.23 & 0 \\
\hline Pto. Baquerizo & S0 53.979 W89 36.558 & 2.56 & 23.5 \\
\hline Pto. Baquerizo & S0 53.911 W89 36.568 & 2.52 & 36 \\
\hline
\end{tabular}

Los datos obtenidos muestran información valiosa respecto del comportamiento del tsunami al momento de impactar las islas, y serán utilizados para validar los resultados obtenidos con los modelos propuestos en el presente estudio

\section{PRONÓSTICO DE CORTO PLAZO}

El pronóstico a corto plazo, pretende estructurar las metodologías del ComMIT/ MOST, a fin de elaborar un modelo que pueda ser utilizado en la modelación de tsunamis, inmediatamente después de conocer su generación. Este modelo, puede ser utilizado tanto en el modo manual como de solución numérica para la combinación de las unidades fuentes. El diseño del pronóstico de corto plazo requiere que se optimicen los modelos en cuanto a rapidez y precisión, para lo cual se debe encontrar la mejor combinación de resolución y extensión de las áreas de estudio[12]. Es importante además que se validen los resultados con datos levantados de eventos históricos, para el presente caso se usaron los datos del Tsunami de Japón 2011.

A diferencia de la metodología propuesta por Tang et al., (2009), en este estudio se prescinde del modelo de referencia, ya que los sitios de interés tienen poca extensión geográfica, por lo consiguiente es posible tener una grilla de alta resolución (15 metros) sin sacrificar el tiempo de cómputo.

La calibración del modelo está en función de obtener un tiempo aceptable en la ejecución y la identificación de los errores de truncado. Para el presente estudio, una de las causas, de errores de truncamiento, fue el coeficiente de Manning, cuya determinación consistió en si misma en un verdadero reto, ya que no se tiene estudios relacionados en el área. Así mismo, se comprobó, que el run-up obtenido era el parámetro más sensitivo a los cambios de valores escogidos para el coeficiente de Manning, tal como fue observado en [12]. También, se pudo constatar que este coeficiente no genera mayor impacto en los registros de mareogramas sintéticos. 
El desarrollo de los modelos de Pronóstico de corto plazo, inicia con el diseño de las grillas a ser utilizadas como dominio computacional para el modelamiento numérico. Estas grillas fueron elaboradas en base a modelos digitales de terreno obtenidas de GMRT(Global Multiresolution Topography)[16], cartas topográficas del Instituto Geográfico Militar, Cartas Náuticas del Instituto Oceanográfico de la Armada, y de una compilación Batimétrica realizada por William Chadwick de la Universidad de Oregón. Todas estas fuentes de datos espaciales fueron referenciadas de manera vertical y horizontal a un mismo nivel de referencia. A fin de considerar el peor escenario, los datos de nivel del mar fueron referenciados a la máxima marea, es decir, la línea de costa. Se utilizaron además dos métodos de interpolación, de acuerdo a la distribución espacial de los datos, estos métodos fueron "Krigging" y "Natural Neighbor". Las grillas obtenidas guardan una relación 1:6 en su resolución, de acuerdo a la recomendación para el modelo MOST; siendo la mas grande en extensión de 18 arcsec $\sim 540$ metros, la mediana de 3 arcsec $\sim 90$ metros y la más pequeña de $0.5 \operatorname{arcsec} \sim 15$ metros de resolución.

La fuente sismo-generadora, fue extraída del ComMIT, la misma que consiste de la combinación lineal de seis unidades fuentes cercanas al epicentro, la misma que se entiende es la mejor combinación obtenida para la simulación del evento en particular, la combinación de las unidades fuentes se aprecia en la Tabla 2.

\begin{tabular}{|l|c|}
\hline Unidad fuente & Slip(m) \\
\hline ki24b & 4.66 \\
\hline ki25b & 12.23 \\
\hline ki26a & 26.31 \\
\hline ki26b & 21.27 \\
\hline ki27a & 22.75 \\
\hline ki27b & 4.98 \\
\hline
\end{tabular}

Tabla 2

Ya que se desconoce el valor de Manning para cada sitio, fue necesario realizar una calibración inicial de este coeficiente, para lo cual se realizaron doce modelamientos, variando los valores del coeficiente desde 0.001 a 0.03 .
Esta validación se realizó de manera cuantitativa y en base de la información disponible. Para casos en los que solamente se cuenta con los valores del levantamiento de la Tabla 1, se realizó una comparación de la extracción de los valores obtenidos del modelo en cada uno de esos puntos. Para los valores del levantamiento se adicionó un margen de error de +-30 centímetros, los cuales son atribuibles al instrumento y el tipo de medición[14]. Se considera que los valores de run-up extraídos del modelo numérico que se encuentren dentro de ese margen, son aceptables.

Así mismo se utiliza la información mareográfica disponible para realizar la validación; para este caso se utilizó el método de Taylor, que valora la capacidad de los modelos para reproducir tanto los valores a comparar como el comportamiento estadístico de la muestra de resultados [17], y facilita la comparación entre varios modelos y los datos de referencia.

En las validaciones se ha considerado que pueden existir diferencias entre lo modelado y los datos reales de referencia, por varias razones entre las que se puede mencionar, la falta de batimetría y topografía de alta resolución, la asumpsion que la propagación del tsunami es lineal, y por el esquema numérico empleado por el modelo[9].

\section{A. PUERTO AYORA}

Para el caso de Puerto Ayora, los valores del coeficiente de Manning que mejor se ajustaron a los valores obtenidos por el levantamiento de Lynett et al., (2011) fueron: 0.015, 0.017, 0.019, 0.020 .

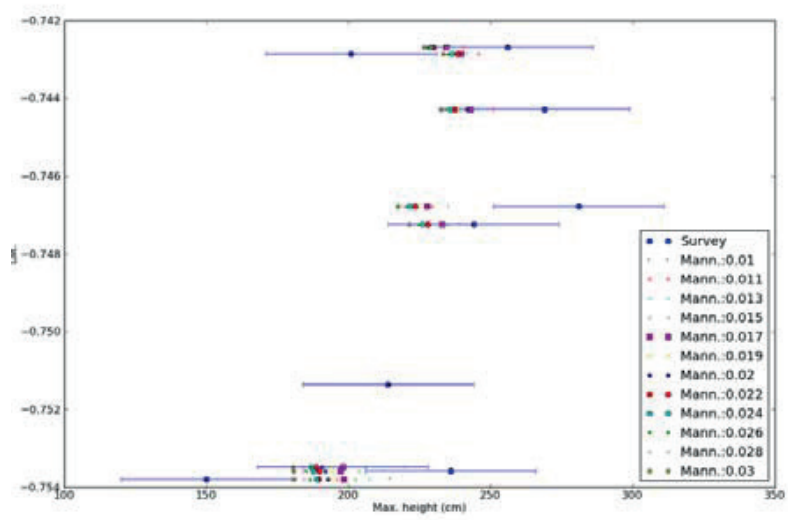

Figura 2. Validación de doce modelos (12 valores de Manning) con los datos levantados por Lynett et al.,(2011). 
De la misma forma se realizó una validación de los doce (12) modelos con el registro mareográfico de la estación ubicada en Puerto Ayora-isla Santa Cruz.

Dado que el método de Taylor valora la correlación entre los datos del modelo y los datos de referencia, fue necesario introducir una compensación en el retardo de la señal del tsunami sintético, hasta conseguir la mejor correlación positiva entre le modelo numérico y los datos de referencia.
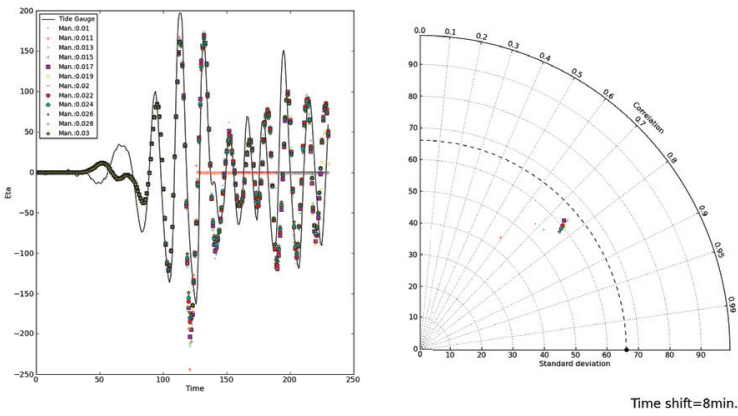

Figura 3. Validación de modelos en base al diagrama de Taylor, para Puerto Ayora.

El valor de Manning escogido fue de 0.019, el mismo que otorgaba una desviación estándar de 60.43 y una correlación de $76 \%$, además se encontró que la señal sintética se encuentra retrasada alrededor de 8 minutos, las causas son atribuibles a las encontradas por [9], la dispersión del tsunamis por su viajes de largas distancias.

El resultado de la máxima inundación para Puerto Ayora, se aprecia en la Figura 4. El modelo de inundación muestra alturas de ola entre 2.3 y 2.6 metros, valores que fueron similares a los encontrando por el levantamiento de [14].

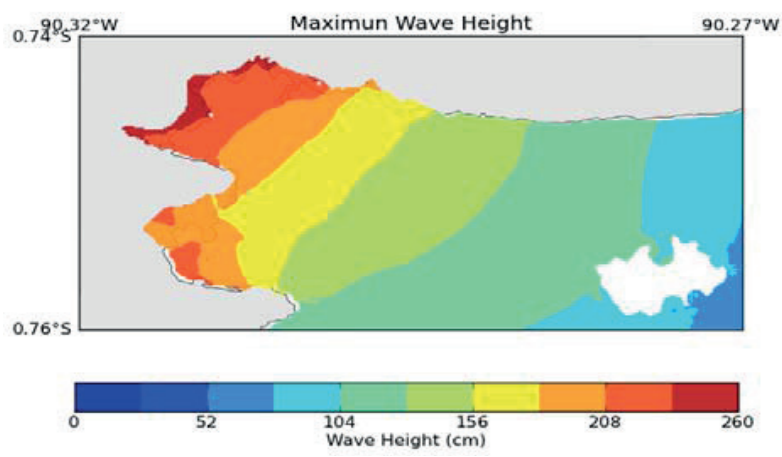

Figura 4. Máxima inundación simulada para Puerto Ayora, Isla Santa Cruz.

\section{B. PUERTO SEYMOUR}

Para el caso de Puerto Seymour no contó con datos de levantamiento, por tal motivo se emplearon los datos del mareógrafo instalado en este sitio. El valor del coeficiente de Manning fue escogido de acuerdo al Método de Taylor, utilizando los mareogramas sintéticos obtenidos con cada modelamiento y comparando estas series con los datos del mareógrafo.

De los resultados obtenidos en cada Modelo se obtuvo que el mejor valor de Manning fue de 0.020 , así mismo se encontró una demora de 13 minutos entre la señal del tsunami sintético y el registro mareográfico.
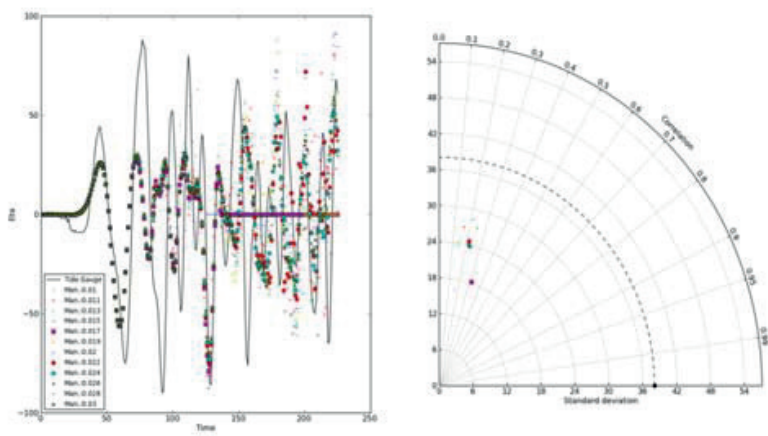

Figura 5. Validación de modelos en base al diagrama de Taylor, para Puerto Seymour.

Con el coeficiente de 0.020 , se simuló el tsunami de Tohoku, obteniendo un mapa de inundación, que se muestra en la Figura 6. Los resultados de este modelo, no pueden compararse con datos reales de inundación, puesto que para este sitio, no se obtuvo esta información.

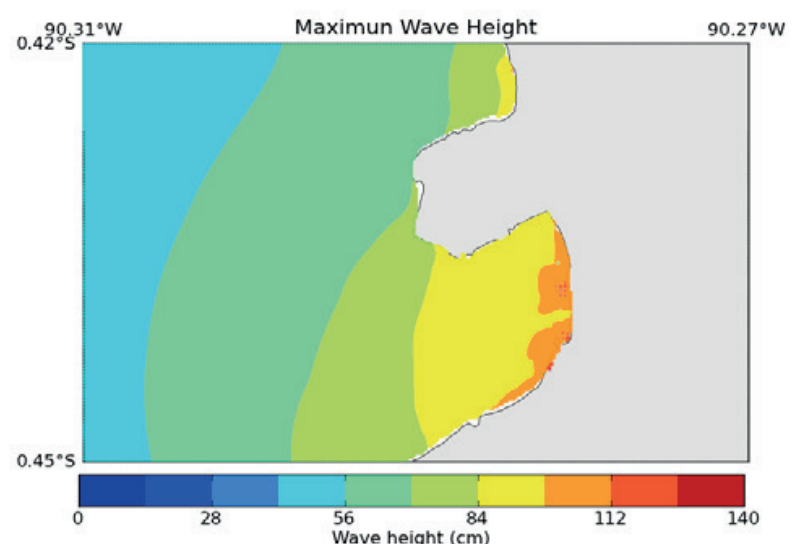

Figura 6. Máxima inundación simulada para Puerto Seymour, Isla Baltra. 


\section{PUERTO BAQUERIZO MORENO}

En el caso de Puerto Baquerizo Moreno, no se contó con registro mareográfico, por tal razón la validación solo puede realizarse por medio de los datos levantados de la Tabla 1.

Los datos obtenidos en estos modelos, mostraron una pobre correlación, por lo que se decidió utilizar un valor de Manning acorde a los valores obtenidos en los casos de Puerto Seymour y Puerto Ayora, el valor escogido fue de 0.02, independiente de la similitud de los datos obtenidos por el modelo en comparación con los datos levantados.

Esta diferencia entre los datos es posiblemente atribuible a la falta de batimetría de alta resolución alrededor de la Isla San Cristóbal. En el modelamiento, se pudo observar que previo a su arribo al Puerto Baquerizo Moreno, las olas interactuaron con el borde oeste de la isla. Esto permite concluir que, si no existe una cobertura batimétrica de alta resolución, como en este caso, esto induce ciertos errores, puesto que esta interacción no es modelada adecuadamente. Por otro lado, el modelo es "no dispersivo", lo que hace que no se incluyan variaciones en la forma de las ondas cuando se propagan en el océano profundo. Nótese en la Figura 7, que existe una diferencia de un metro aproximadamente entre los datos modelados con los datos levantados.

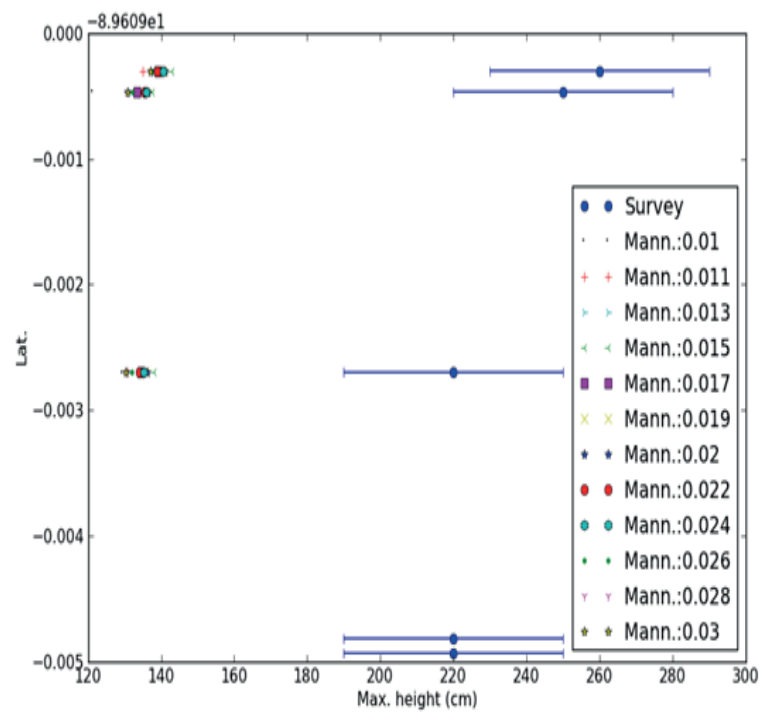

Figura 7. Validación de los modelos en base a los datos de la Tabla 1, en Puerto Baquerizo Moreno.
Con un coeficiente de Manning de 0.02, se realizó la simulación en el Puerto Baquerizo Moreno, obteniendo los resultados mostrados en la Figura 8.

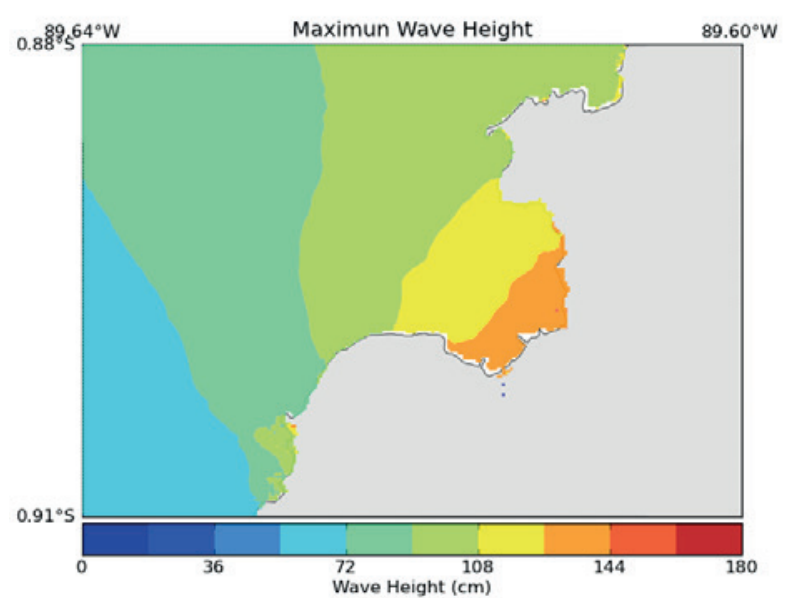

Figura 8. Máxima inundación modelada en Pto. Baquerizo Moreno, debido al Tsunami de Tohoku.

\section{PRONÓSTICO DE LARGO PLAZO}

El pronóstico de largo plazo se realiza para Puerto Seymour, Puerto Ayora y Puerto Baquerizo Moreno, y tuvo por objetivo la identificación de las áreas de mayor potencial para producir tsunamis que puedan afectar a los sitios de estudio.

Se realizó la simulación de tsunami sintético a lo largo de la costa del Pacifico, realizando diferentes combinaciones lineales de la fuente de manera de obtener un tsunami de magnitud 8.5 , es decir suficientemente fuerte como para garantizar el arribo de las perturbaciones hasta Galápagos.

Se llevaron a cabo un total de 104 modelamiento numéricos, con escenarios formados por la combinación de 6 unidades fuentes y un slip uniforme de 5.26 metros, a fin de obtener una magnitud simulada de $8.5 \mathrm{Mw}$.

La extracción de los máximos para cada sitio se obtuvo del mareograma sintético tomado de cada modelo, y en la posición de los mareógrafos de los sitios Puerto Ayora y Puerto Seymour.

Las zonas identificadas como de potencial 
peligro para Puerto Ayora fueron: la zona de subducción de la Aleutianas, la zona de subducción Ecuador-Colombia y la zona de subducción de Nueva Zelanda-KermadecTonga.

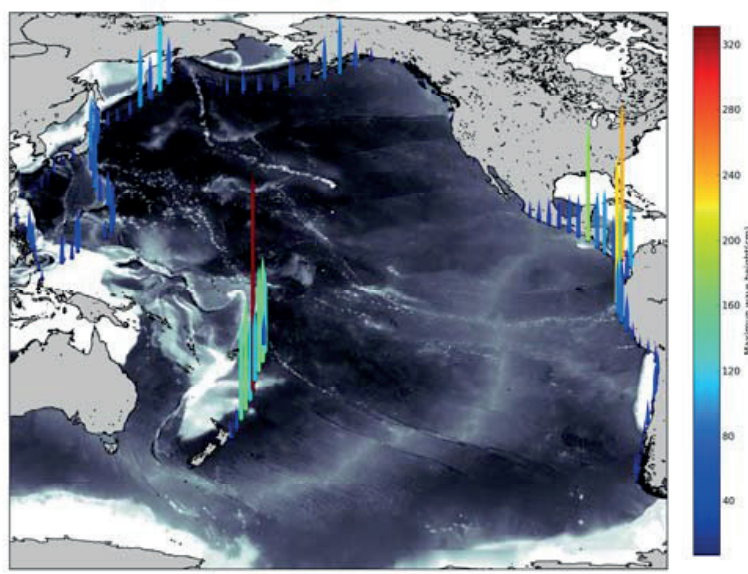

Figura 9. Pronóstico de largo plazo para Puerto Ayora.

Considerando las máximas inundaciones producidas por cada uno de estos 104 modelamientos, se obtuvo el máximo de los máximos de inundación, a fin de obtener las áreas inundable en Puerto Ayora, con la consideración de un sismo-tsunami de $8.5 \mathrm{Mw}$ en cualquier lugar del Océano Pacifico. El mapa resultado se aprecia en la Figura 10.

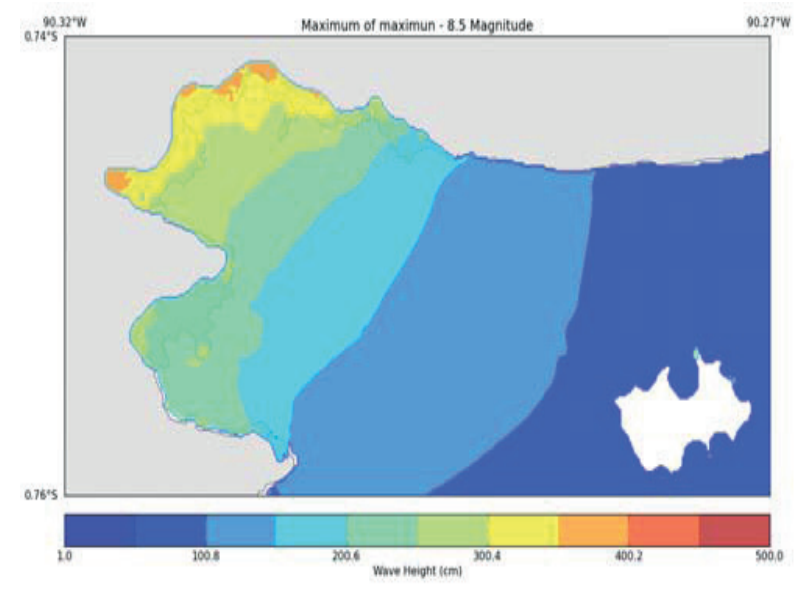

Figura 10. Máxima inundación para Puerto Ayora, en el escenario múltiple de 104 tsunamis.

Se considera que este mapa puede ser utilizado para propósitos de gestión de riesgos como mapa de inundación para el diseño de rutas de evacuación y planes de contingencia, ya que abarca un escenario múltiple de generación de tsunamis.
De igual forma se modelaron los efectos de los 104 tsunamis en el Puerto Seymour. Se identificó que el mayor peligro potencial lo representan las zonas de subducción de América Central y la de Ecuador-Colombia.

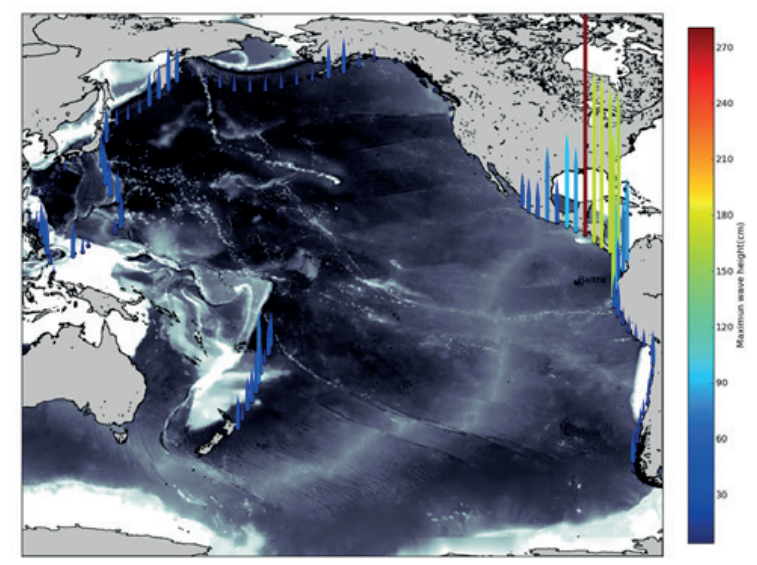

Figura 11. Pronóstico de largo plazo para Puerto Seymour

Finalmente, se realizó el modelamiento para el Puerto Baquerizo Moreno, identificándose que, la amenaza potencial se encuentra localizada en las zonas de subducción de América Central y Ecuador-Colombia. Es decir, tiene una similitud con la de Puerto Seymour.

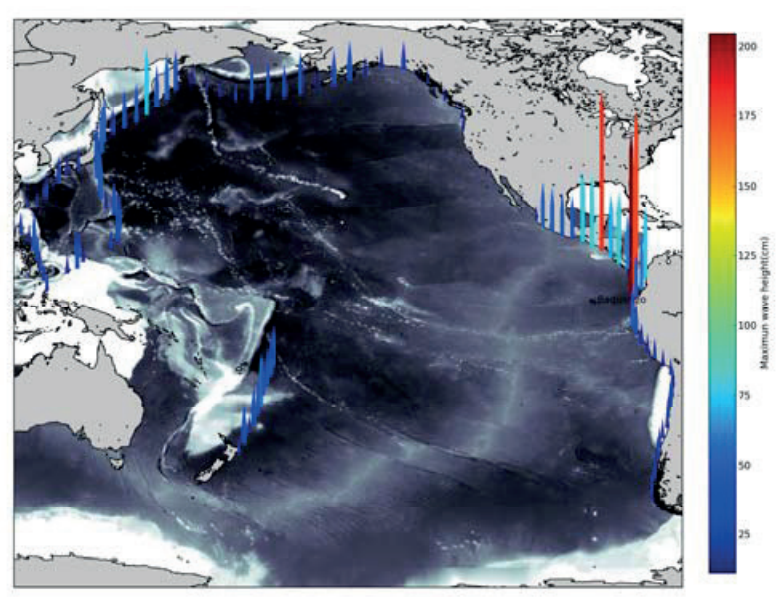

Figura 12. Pronóstico de largo plazo para el Puerto Baquerizo Moreno

La máxima inundación debida a los 104 modelamientos se representa en la Figura 13, aquí se observa que los máximos de inundación esperados por un sismo-tsunami de $8.5 \mathrm{Mw}$, se encuentran en la zona central de la bahía. Es necesario destacar que esta altura de inundación se mide desde la máxima marea. 


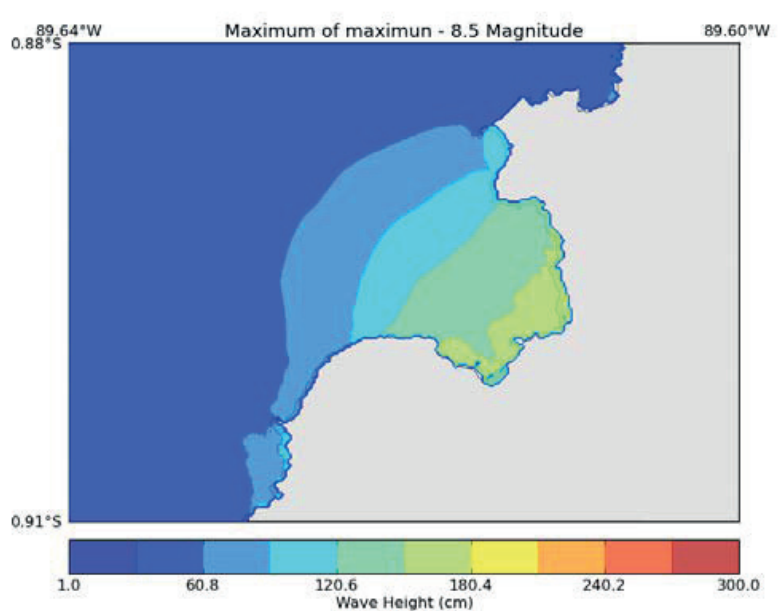

Figura 13. Máxima inundación para Puerto Baquerizo Moreno, en el escenario múltiple de 104 tsunamis.

\section{CONCLUSIONES}

Los resultados obtenidos en la obtención del pronóstico de corto plazo, indican la necesidad de una mejor calibración de las grillas utilizadas para el modelamiento. Es necesario realizar un estudio más detallado para obtener los mejores modelos digitales de terreno y utilizarlos nuevamente en los modelamientos. La ventaja de la metodología, es que una vez que se tenga una grilla con datos de mejor calidad, esta puede ser remplazada, tomando en consideración que la extensión y la resolución deben ser las mismas.

Así mismo se destaca la necesidad de comparar los resultados con el empleo de otros modelos numéricos. Es necesario que se considere la dispersión física de las olas al propagarse grandes distancias, y verificar si existen diferencias en los efectos del impacto de las olas. Estas observaciones sin embargo no limitan la capacidad del modelo para ser utilizada en el pronóstico operacional y ser utilizado para los fines descritos en este estudio, ya que los resultados para Puerto Ayora, demostraron una buena capacidad para representar la realidad y el modelo ha sido ampliamente utilizado y validado en otros sitios. Es importante destacar, la rigurosidad de los métodos de comparación empleados en este estudio. Los mismos que exigen una buena correlación de los datos modelados con los datos reales. Por tal motivo, los resultados obtenidos en el pronóstico de corto plazo, pueden ser utilizados para la elaboración de planes de respuesta y para orientar a la población a reaccionar y evacuar de lugares de riesgo.

Los resultados obtenidos en el pronóstico de largo plazo, pueden ser utilizados en la gestión del riesgo de tsunamis, con la identificación de las zonas de mayor peligro para cada sitio, y como modelos de referencia para cuantificar y comparar los efectos del tsunami, cuando este ocurra.

\section{AGRADECIMIENTOS}

A los profesores Patrick Lynett y Robert Weiss por la asistencia brindada en la investigación de este tema. Al Prof. Robert Hetland por incentivarme a escribir este artículo.

El presente estudio se realiza como parte del Proyecto de Maestría en Ciencias en Oceanografía realizado en Texas A\&M University, gracias a la beca concedida por el Convenio INOCARSENESCYT.

\section{REFERENCIAS}

[1] V. V. Titov, F. I. Gonzalez, and P. M. E. L. (U.S.), Implementation and Testing of the Method of Splitting Tsunami (MOST) Model. U.S. Department of Commerce, National Oceanic and Atmospheric Administration, Environmental Research Laboratories, Pacific Marine Environmental Laboratory, 1997.

[2] V. V. Titov, C. W. Moore, D. J. M. Greenslade, C. Pattiaratchi, R. Badal, C. E. Synolakis, and U. Kânoğlu, "A New Tool for Inundation Modeling: Community Modeling Interface for Tsunamis (ComMIT)," Pure and Applied Geophysics, vol. 168, no. 11, pp. 2121-2131, Mar. 2011.

[3] V. V. Titov, F. I. Gonzalez, E. N. Bernard, 
M. C. Eble, H. O. Mofjeld, J. C. Newman, and A. J. Venturato, "Real-Time Tsunami Forecasting: Challenges and Solutions," Natural Hazards, vol. 35, no. 1, pp. 35-41, 2005.

[4] V. V. Titov and C. E. Synolakis, "Numerical Modeling of Tidal Wave Runup," Journal of Waterway, Port, Coastal, and Ocean Engineering, vol. 124, no. 4, pp. 157-171, Jul. 1998.

[5] V. V. Titov and C. E. Synolakis, "Modeling of Breaking and Nonbreaking Long-Wave Evolution and Runup Using VTCS-2," Journal of Waterway, Port, Coastal, and Ocean Engineering, vol. 121, no. 6, pp. 308316, Nov. 1995.

[6] N. N. Yanenko, The Method of Fractional Steps: The Solution of Problems of Mathematical Physics in Several Variables, 1st ed. Springer, 1971.

[7] Y. Okada, "Surface Deformation Due to Shear and Tensile Faults in a Half-Space," Bulletin of the Seismological Society of America, vol. 75, no. 4, pp. 1135-1154, Aug. 1985.

[8] Y. Wei, E. N. Bernard, L. Tang, R. Weiss, V. V. Titov, C. Moore, M. Spillane, M. Hopkins, and U. Kânoğlu, "Real-time experimental forecast of the Peruvian tsunami of August 2007 for U.S. coastlines," Geophysical Research Letters, vol. 35, no. 4, Feb. 2008.

[9] D. B. Percival, D. W. Denbo, M. C. Eblé, E. Gica, H. O. Mofjeld, M. C. Spillane, L. Tang, and V. V. Titov, "Extraction of tsunami source coefficients via inversion of DART buoy data," Natural Hazards, vol. 58, no. 1, pp. 567-590, Dec. 2010.

[10] S. L. Douglas, "Estimating Runup on Beaches: A Review of the State of the Art," Oct. 1990.

[11] P. L. F. Liu, Advanced Numerical Models for Simulating Tsunami Waves and Runup. World Scientific, 2008.
[12] L. Tang, V. V. Titov, and C. D. Chamberlin, "Development, testing, and applications of site-specific tsunami inundation models for real-time forecasting," Journal of Geophysical Research, vol. 114, no. C12, Dec. 2009.

[13] P. L. F. Liu, in Tsunamis, E. N. Bernard and A. R. Robinson, Eds. Harvard University Press, 2009, pp. 295-319.

[14] P. Lynett, R. Weiss, W. Renteria, G. Morales, and S. Son, "Coastal Impacts of the March 11th Tsunami in the Galapagos Islands," no. Submitted, 2011.

[15] P. Lynett, J. Borrero, S. Son, R. Weiss, D. Greer, and W. Renteria, "Observations and Modeling of Tsunami-Induced Currents in Ports and Harbors," no. Submitted, 2011.

[16] W. B. F. Ryan, S. M. Carbotte, J. O. Coplan, S. O'Hara, A. Melkonian, R. Arko, R. A. Weissel, V. Ferrini, A. Goodwillie, F. Nitsche, J. Bonczkowski, and R. Zemsky, "Global Multi-Resolution Topography synthesis," Geochem. Geophys. Geosyst., vol. 10, p. 9 PP., Mar. 2009.

[17] K. E. Taylor, "Summarizing multiple aspects of model performance in a single diagram," J. Geophys. Res., vol. 106, no. D7, pp. PP. 7183-7192. 